\title{
Acknowledgement to Reviewers of $C$ in 2017
}

\section{$C$ Editorial Office}

MDPI AG, St. Alban-Anlage 66, 4052 Basel, Switzerland

Published: 12 January 2018

Peer review is an essential part in the publication process, ensuring that $C$ (Journal of Carbon Research) maintains high quality standards for its published papers. In 2017, a total of 37 papers were published in the journal. Thanks to the cooperation of our reviewers, the median time to first decision was 16 days and the median time to publication was 39 days. The editors would like to express their sincere gratitude to the following reviewers for their time and dedication in 2017:

\author{
Adelhelm, Philipp \\ Agostini, Marco \\ Alegre, Cinthia \\ Alvarez, Noe T. \\ Amiri, Ali \\ Andreoli, Enrico \\ Anyszka, Rafał \\ Ayres, Robert U. \\ Barnes, Frank S. \\ Bashkova, Svetalana \\ Battistel, Alberto \\ Borondics, Ferenc \\ Bowen, Chris R. \\ Calabrò, Francesco \\ Campbell, Patrick \\ Campuzano Ruiz, Susana \\ Carabineiro, Sonia A. C. \\ Cazorla, Claudio \\ Chen, Binling \\ Chen, Chunhui \\ Chen, Ruiyong \\ Chiolerio, Alessandro \\ Corry, Ben \\ Cuartero, María \\ Dang, Alei \\ David, Lamuel \\ De La Escosura, Alfredo \\ Debéda, Hélène \\ Dickert, Franz L.
}

\author{
Dimitratos, Nikolaos \\ Dinesh, Bhimareddy \\ Dorri Moghadam, Afsaneh \\ Duong, Hai Minh \\ Dyatkin, Boris \\ Enyashin, Andrey \\ Fan, Ruiqing \\ Feng, Yunlong \\ Fujiwara, Toshimichi \\ Gao, Weilu
}

Girousi, Stella

Gomes, João Fernando Pereira

Guo, Yuanhao

Guo, Zhanhu

Güttel, Robert

Hayami, Wataru

Hayashi, Akari

Hiete, Michael

Higuchi, Eiji

Huang, Peihua

Iannazzo, Daniela

Ikeda, Atsushi

Islam, Mahbubul

Janegitz, Bruno Campos

Jena, Umakanta

Jung, Kwang Deog

Khan, Amir

Kichambare, Padmakar

Kiciński, Wojciech 
Kim, Jin Young

Kim, Myung Jun

Kim, Yoong

Konarev, Dmitri

Kopel, Pavel

Koskinen, Pekka

Lazik, Detlef

Le, Duy

Lee, Byung Yang

Lemeune, Alla

Lemus, Jesús

Lin, Fei

Liu, Tianyu

Love, Jenny

Macario, Anastacia

Mäki-Arvela, Päivi

Martone, Alfonso

Matei Ghimbeu, Camelia

Meille, Valérie

Melchionna, Michele

Mengistie, Desalegn Alemu

Menzel, Robert

Mohammad, Arjmand

Nagai, Hiroki

Nakanishi, Ryo

Nishiyama, Yusuke

Oschatz, Martin

Otomo, Junichiro

Palmer, Jeremy C.

Park, Joon-Shik

Park, Min-Sik

Patil, Devendra

Peampermpool, Rakpong

Pérez, Esther Enríquez

Phung, Quoc Tri

Polanski, Marek

Radecki, Jerzy

Ragauskas, Arthur

Robles, Guillermo
Roger, Leblanc

Romar, Henrik

Rosa, Rui Namorado

Rudykh, Stephan

Ryan, James G.

Ryu, Won-Hee

Schweiss, Ruediger

Sharifi, Tiva

Shimoi, Norihiro

Shirvanimoghaddam, Kamyar

Shokouhimehr, Mohammadreza

Shrestha, Lok Kumar

Smått, Jan-Henrik

Sockalingam, Subramani

Sohgawa, Masayuki

Song, Kenan

Song, Young Jae

Su, Ming-Der

Sun, Hongyu

Szekely, Gyorgy

Tabish, Tanveer

Taddei, Maurizio

Taffese, Woubishet Zewdu

Tagliatesta, Pietro

Tamminen, Tarja

Teymourian, Hazhir

Thakur, Vijay Kumar

Tobias, Gerard

Tomita, Shunsuke

Vautard, Frederic

Venditti, Iole

Wang, Tanyu

Wang, Xiaohan

Xia, Guanglin

Yoshitake, Hideaki

Yurchenko, Daniil

Zhai, Xianglin

Zhao, Bote 